\title{
Nonextensive Dynamics of Drifting Sea Ice
}

\author{
Alexandre Chmel, ${ }^{1}$ and Victor Smirnov ${ }^{2}$ \\ ${ }^{1}$ Fracture Physics Department, Ioffe Physico-Technical Institute, Russian Academy of Sciences, St. Petersburg 194021, Russia \\ ${ }^{2}$ Arctic and Antarctic Research Institute, 38 Bering Street, St. Petersburg 199397, Russia \\ Correspondence should be addressed to Alexandre Chmel; chmel@mail.ioffe.ru
}

Received 30 July 2012; Accepted 8 October 2012

Academic Editors: M. Lipinski and J. L. Zhou

Copyright (c) 2013 A. Chmel and V. Smirnov. This is an open access article distributed under the Creative Commons Attribution License, which permits unrestricted use, distribution, and reproduction in any medium, provided the original work is properly cited.

Cycles of ice pack fragmentation in the Arctic Ocean are caused by the irregular drift dynamics. In February 2004, the Russian ice-research camp North Pole 32 established on a floe in the Arctic Ocean ceased its working activity and was abandoned after a catastrophic icequake. In this communication, the data collected during the last month of the field observations were used for calculating the changes in the kinetic energy of the ice floe. The energy distribution functions corresponding to periods of different drift intensity were analyzed using the Tsallis statistics, which allow one to assess a degree of deviation of an open dynamic system, such as the drifting ice, from its equilibrium state. The obtained results evidenced that the above-mentioned critical fragmentation has occurred in the period of substantially nonequilibrium dynamics of the system of ice floes. The determination of the state of the pack (in the sense of its equilibrium/nonequilibrium) could provide some useful information on forthcoming icequakes.

\section{Introduction}

From the viewpoint of conventional mechanics, the Arctic sea ice cover (ASIC) is the consolidated, mobile, deformable system. Prevalently shearing deformations result in regular pattern of fragmented pack in accordance with the Mohr's mechanism of semibrittle failure of solids.

At the same time, the size distribution of sea ice floes does not exhibit the random (Poissonian-like) statistics and follows the power law typical for scaling (fractal) structures $[1,2]$. The conventional mechanics cannot explain this phenomenon.

The scaling is a manifestation of long-range correlations between separated events in a statistical system. The correlation radius is determined by the spatial decay of the event effect. The decaying is fast (exponential) in equilibrium systems but slow (power law) in nonequilibrium ones. Therefore, the fractal structures are formed only under nonequilibrium conditions in statistical systems driven by external forcing.

In recent years, a new statistical concept called the nonextensive statistical mechanics (NESM) was developed by Tsallis $[3,4]$ for thermodynamic description of the behavior of multiscale systems revealing fluctuations around their equilibrium state. The NESM was successively applied for assessing the degree of deviation of natural dynamic systems from their equilibrium state prior to large-scale hazards, such as earthquakes $[5,6]$ and floods [7]. In the ASIC, cycles of pack fragmentation and significant icequakes occur due to irregularity in the ice drift, which causes critical deformations in the ice cover. According to the NESM, the formation of fractal structures, such as fragmented ice pack, is a result of nonequilibrium dynamics of open systems. In this work, the sea ice drift dynamics during a few bursts of significant fragmentations, which could be regarded as natural hazards, were analyzed in terms of the NESM.

The data on the local drift dynamics were collected in the Russian ice research camp North Pole 32 (NP 32) that was established on ice pack and drifted in 2003-2004 in the region north from Franz Josef Land from $88^{\circ} \mathrm{N}$ to $84^{\circ} \mathrm{N}$ [8]. In this work, the time series of the kinetic energy variation of an individual ice floe were analyzed using the Tsallis statistics in order to obtain a thermodynamic characteristic of the system of drifting ice prior to and during large-scale sea ice fragmentations. 


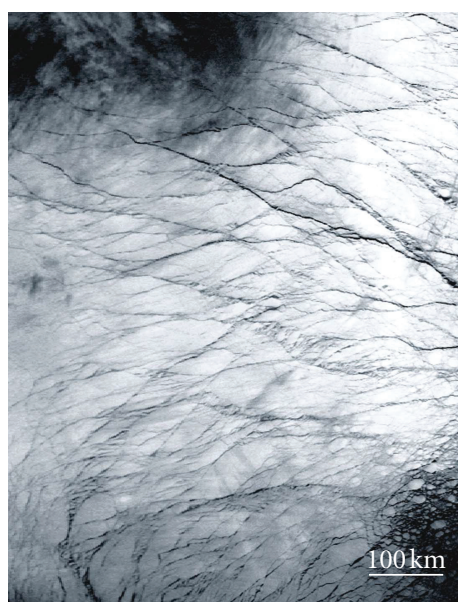

FIGURE 1: AVHRR image taken from NOAA satellite on 6 December 2003 in the region around $82^{\circ} \mathrm{N}, 5^{\circ} \mathrm{E}$.

\section{Geometry and Size}

A distinguishing feature of the Arctic sea-ice cover (ASIC) is a rectilinear lead pattern characterized by the constant angle $\sim 30^{\circ}$ between directions of intersecting leads (Figure 1). This regular structure consists of parallelogram-like figures ranging from 1 to $\sim 10^{2} \mathrm{~km}$. Marco and Thompson [9] explained this phenomenon on the base of the Mohr's theory of failure, which suggests the following relation between the applied shear stress at failure, $\tau$, and the normal stress, $\sigma_{n}$, on the failure plane (or line in $2 \mathrm{D}$-case):

$$
\tau=C+\sigma_{n} \tan \varphi,
$$

where $C$ is the material cohesion strength, and $\varphi$ is the angle of internal friction, $\varphi= \pm\left(\alpha-45^{\circ}\right)$; here $\alpha$ is the intersection angle. The resolution of (1) relatively the intersection angle with parameters characteristic for sea ice gives one $\alpha \approx 30^{\circ}$, that is the value observed in many satellite images (Figure 1).

The parallelogram-like pattern appears only at sufficiently rapid strain rates when the semibrittle failure prevails. At relatively slow strain rates, the ductile behavior takes place, which results in formation of polygonal (diamond-like) or fully disordered structures due to cohesion flow at low yield stress [9].

At the same time, there are some features in the lead pattern, which cannot be explained in the framework of classical mechanics. Rothrock and Thorndike [10] and Matsushita [11] studied the size distribution of sea ice floes and revealed a scale invariant relation between the number of floes and their size in the form

$$
N\left(z>z^{\prime}\right) \propto z^{-\beta},
$$

where $N\left(z>z^{\prime}\right)$ is the number of floes larger than $z^{\prime}$; $z$ is the dimensional characteristics, such as area, $S$, or length, $L$; $\beta$ is the constant. The power law dependence means the selfsimilarity of the ensemble of ice floes because the power law function (2) is the unique solution of the scaling relation

$$
N(\lambda z)=\lambda^{-\beta} N(z),
$$

where $\lambda$ is the constant (scaling factor). In general, the power law behavior is regarded as a representation of the scale invariance of an object or process in space, time, or energy (in dependence of the measured scaling parameter).

The scaling (power law) number-size distributions were found for lead patterns formed both by ice floes of regular geometry, such as parallelogram-like and diamond-like floes, and by "smoothed" (oval) floes typical for frost-free seasons.

Another example of the invariance of the floe geometry was found by Chmel et al. [2] who had shown that the floe area and floe perimeter are interconnected through the following relation:

$$
S \propto L^{2 / D}
$$

The spatial invariance of this kind is characterized by the parameter $D$ called the "fractal dimension", which, as distinct from the integer Euclidian dimension, could take a fractional value. In the "two-dimensional" case, $D$ could take any value from 1 to 2 . Highly fragmented pack with a vast of one dimensional features, such as leads and cracks is characterized by $D$ close to 1 , while the fractal dimension of consolidated, frozen ice fields should be closer to 2 .

Figures 2 and 3 show examples of the power law dependences (2) and (4) calculated for lead-and-crack pattern in the ice cover shown in Figure 1.

The fragmentation of the Arctic ice pack is caused by nonuniform sea ice drift, which is accompanied with substantial stress redistributions. To understand the driving force of self-organizing in fractal structures one should consider the thermodynamic conditions of the drift dynamics.

\section{Nonextensive Dynamics}

The thermodynamic state determines the principal trends in the behavior of every statistical system. The most significant perturbations occur when the system deviates from its equilibrium state. Under nonequilibrium conditions, individual "events" in the system are not independent but affect each other at distances much longer than the size of an excited locality. A substantial effect of local event on adjacent sites disturbs the entropic additivity of the process, which in the simplest case of two independent equilibrium subsystems $A$ and $B$ can be expressed as:

$$
S(A+B)=S(A)+S(B)
$$

where $S$ is the entropy.

The thermodynamic description of processes in nonequilibrium statistical systems was developed by Tsallis [3, 4]. The main idea forwarded in his approach was to introduce into additive expression (5) a cross-member that would take into account some interactions between subsystems through a "parameter of nonextensivity" q:

$$
S(A+B)=S(A)+S(B)+\frac{S(A) S(B)(1-q)}{k} .
$$

Here $k$ is the Boltzmann constant. 


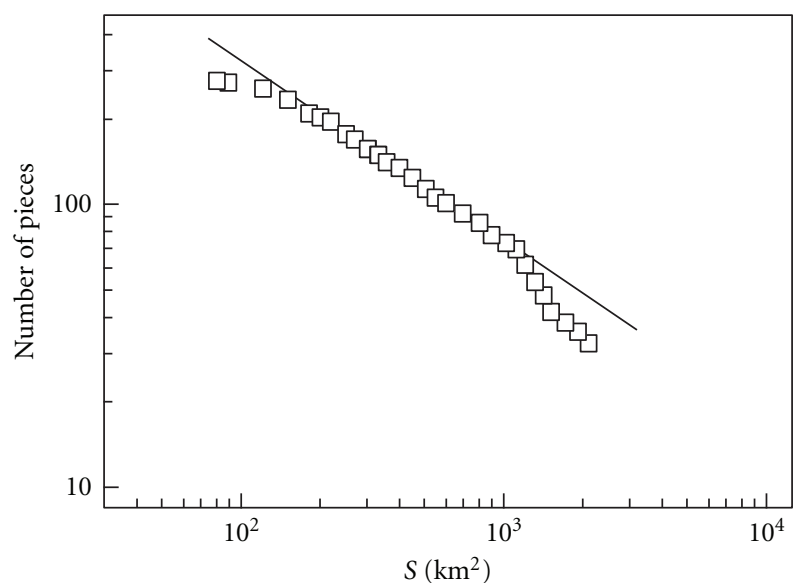

FIGURE 2: Cumulative distribution of floe sizes calculated by processing the satellite image shown in Figure 1. Straight line is the best fitted power law dependence (2).

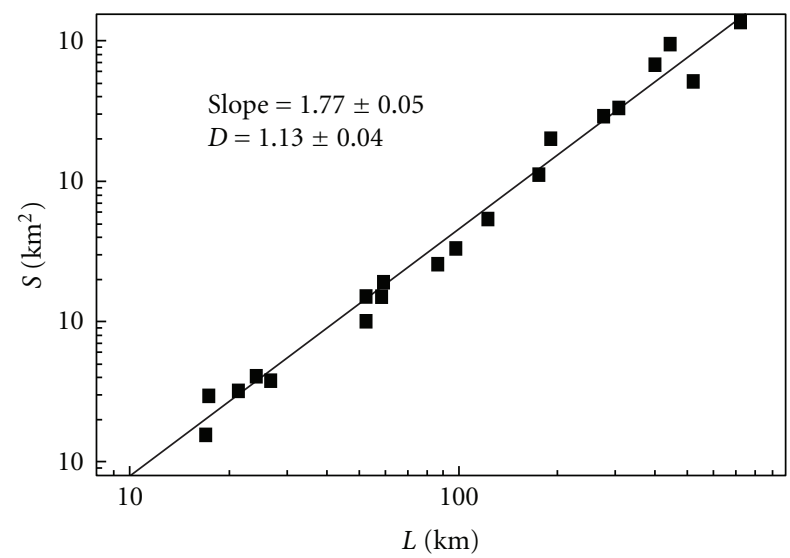

Figure 3: Area versus perimeter dependences for ice-floes fragments resolved in the satellite image shown in Figure 1. The straight line fits the power law dependence (4).

Passing over details of the Tsallis' consideration (see [3, 4] for details), we note that the classical statistical mechanics responds the limit $q \rightarrow 1$ ((6) transforms into (5)); for $q<1$ the formalism imposes a limited number of events in equilibrium system; finally, the value $q>1$ indicates the nonequilibrium state of the dynamic system - this is the case of non-extensive dynamics, which is incompatible with the entropic additivity.

The value of the parameter $q$ serves as a measure of deviation of the statistical system from its equilibrium state. The stronger deviation, the higher $q$. Accordingly, the higher $q$, the larger correlation radius of interactions, and the cooperative effects are more pronounced.

Abe and Suzuki $[12,13]$ used the non-extensive paradigm in their analysis of the distance and waiting time distributions between successive earthquakes and derived a universal expression for the probability of events in nonequilibrium system:

$$
P\left(x>x^{\prime}\right)=\left[1-\frac{(1-q) x^{\prime}}{x_{0}}\right]^{1 /(1-q)},
$$

where $P\left(x>x^{\prime}\right)$ is the probability of being the value $x$ higher than a threshold $x^{\prime}, x_{0}$ is the mean value of $x$ in the series.

Equation (7) was used by Abe and Suzuki for fitting the parameters $q$ and $x_{0}$ to known distances [12] and waiting time [13] distributions between earthquakes. The expression for the cumulative energy distribution $N\left(\varepsilon>\varepsilon^{\prime}\right)$ versus $\varepsilon^{\prime}$ can written in the following form:

$$
N\left(\varepsilon>\varepsilon^{\prime}\right)=P\left(\varepsilon>\varepsilon^{\prime}\right) \times N=N\left[1-\frac{(1-q) \varepsilon^{\prime}}{\varepsilon_{0}}\right]^{1 /(1-q)},
$$

where $N\left(\varepsilon>\varepsilon^{\prime}\right)$ is the number of events satisfying the inequality $\varepsilon>\varepsilon^{\prime}$, and $N$ is the total number of events. Some opportunities given by the applying of (8) in studies of sea ice drift dynamics were demonstrated recently by $\mathrm{Chmel}$ et al. [14].

In this work we used (8) for analyzing the time series of changes in drift speed of an individual ice floe. In this context, an "event" should be regarded as a change in the kinetic energy of the floe; $\varepsilon_{0}$ is the mean value of $\varepsilon$ in a selected time interval.

\section{Energy Exchange}

The energy exchange between a drifting ice floe and its environment can be found from changes in the speed of the floe caused by impact or shear interactions with neighboring floes. The kinetic energy change, $\varepsilon$, is directly proportional to the speed change squared of the ice floe, $\varepsilon \propto \Delta V^{2}$ (where $\Delta V^{2}=\left|V^{\prime 2}-V^{\prime \prime 2}\right|, V^{\prime}$ and $V^{\prime \prime}$ are the ice floe speeds measured in two subsequent time intervals). Therefore, the distribution function, $N\left(\varepsilon>\varepsilon^{\prime}\right)$, is equivalent to the distribution of the values $\Delta V^{2}$.

The time series of changes in the kinetic energy of the ice floe were recorded in the Russian ice research camp NP 32.

In February 2004, a series of icequakes caused the intensive ice cover fragmentation in the region of the NP 32 activity. The GPS monitoring of the camp position showed a series of local pack displacements with the most intensive shocks on 18 February. In addition, multiple fragmentations of the ice floe, on which the NP 32 drifted, were detected as beginning from the day of the main lead propagation and during subsequent days of February. As a result, the scientific observations were ceased on 26 February; on 2 March 2004 the research station NP 32 was abandoned in connection with multiple breakage of the ice floe. The time series of the floe kinetic energy changes were now used for assessing the disequilibrium of the pack state prior to, during, and after the occurred icequake.

The values $V$ were found from the changes in the geopositioning data determined by a GPS transducer. The 


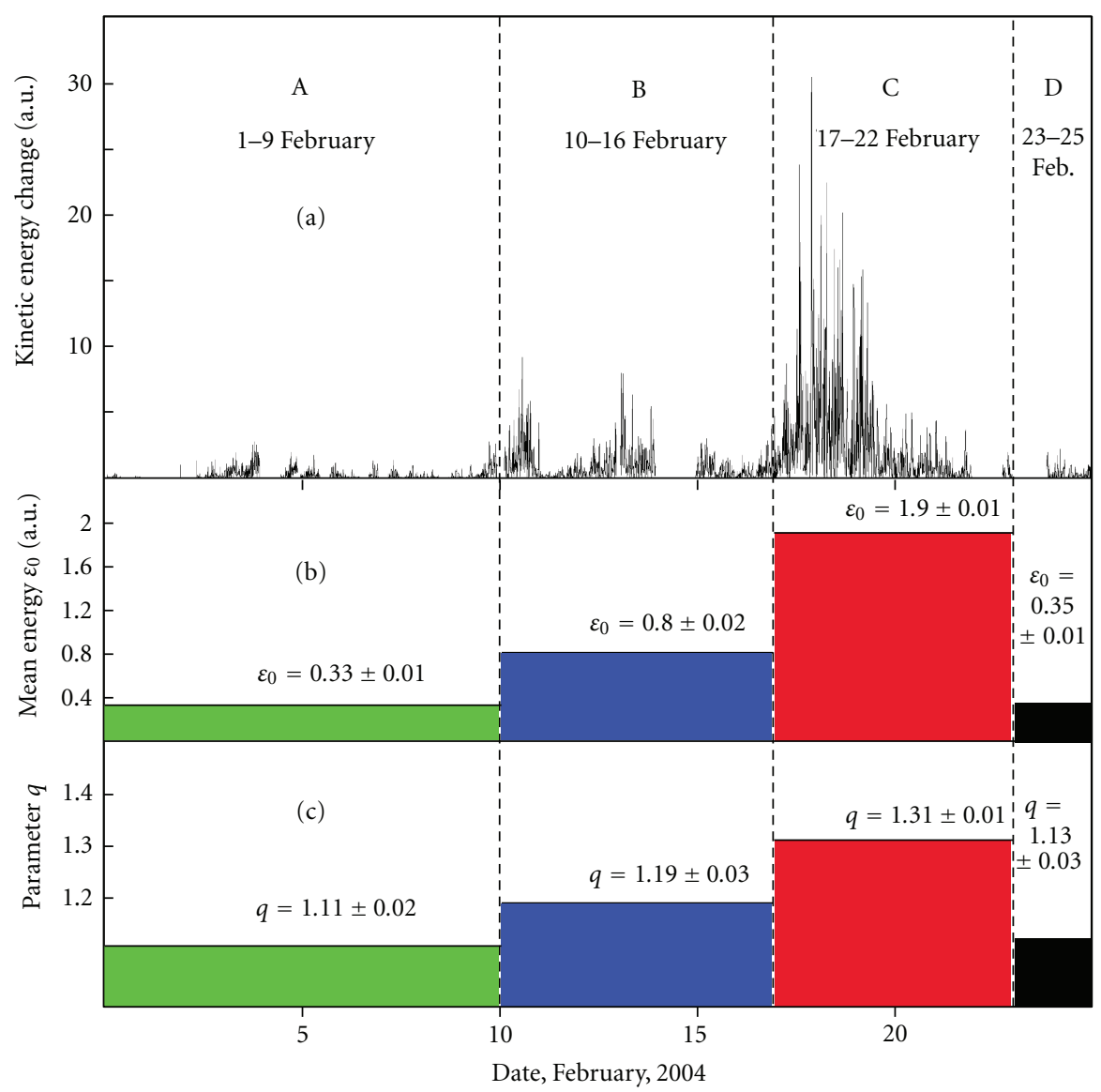

FIGURE 4: (a) Amplitudes of kinetic energy changes found from the ice floe speed data in the time interval 1 February to 26 February 2004 . A few gaps are due to instrumental failures caused by local breakings in the ice floe. (b) and (c) Histograms of the mean value of kinetic energy change $\varepsilon_{0}$ and the parameter of nonextensivity $q$, respectively.

changes in the ice floe speed were measured in 10 minutes intervals. The measurement procedure and the accuracy of the determination of changes in the value of the ice floe speed using GPS transducer are given in [15]. A time series of kinetic energy changes (proportional to the measured $\Delta V^{2}$ values) in the time period of drift from 1 February to 26 February 2004 is depicted in Figure 4(a). The whole period of observations was divided in four "time windows" in accordance the varied intensity of floe displacements in different periods of time.

Figure 5(a) shows the energy distributions $N\left(\varepsilon>\varepsilon^{\prime}\right)$ versus $\varepsilon^{\prime}$ in each time window. The measured distributions were approximated by the function (8) using the procedure of best fitting applied to the parameters $\varepsilon_{0}$ and $q$. The calculated analytical curves are shown in Figure $5(\mathrm{a})$ by lines. The found best-fitted values of the mean value of the energy change and the parameter of nonextensivity are shown as histograms in Figures 4(b) and 4(c), respectively. One can see that the parameter $\varepsilon_{0}$ (Figure $4(\mathrm{~b})$ ) reproduces adequately the intensity of ice floe mobility (Figure $4(\mathrm{a})$ ) in different time intervals. In a similar way, the $q$-value is minimal in the window $\mathrm{A}$ that is prior to the beginning of the sea ice fragmentation. Its closeness to unity evidences the almost equilibrium state of the drifting sea ice. In the subsequent interval B characterized by the moderate ice floe mobility, the $q$-value increased from 1.11 to 1.19 thus indicating an increased deviation of the ice pack from the equilibrium state. The highest value of the parameter $q$ (1.31) was achieved in window $\mathrm{C}$ when the largest changes in the floe kinetic energy were recorded. At the final stage of observations (window D), the value $q$ returned to its initial value.

The variation of the $q$-value reflects trends in the behavior of ice pack influenced by the external forcing. A deviation of the parameter $q$ from unity means the nonequilibrium state of drifting sea ice. Under this condition, the fragmentation of the pack would tend to form fractal structures.

\section{Discussion}

Classical statistical mechanics are constructed on the implication of the independence of individual events involved in the mechanical process; each event produces an additive contribution. The additivity (extensivity) of this kind is the basic property of closed systems.

The mechanical behavior of open, nonequilibrium thermodynamic systems, such as the ASIC, is affected by the 


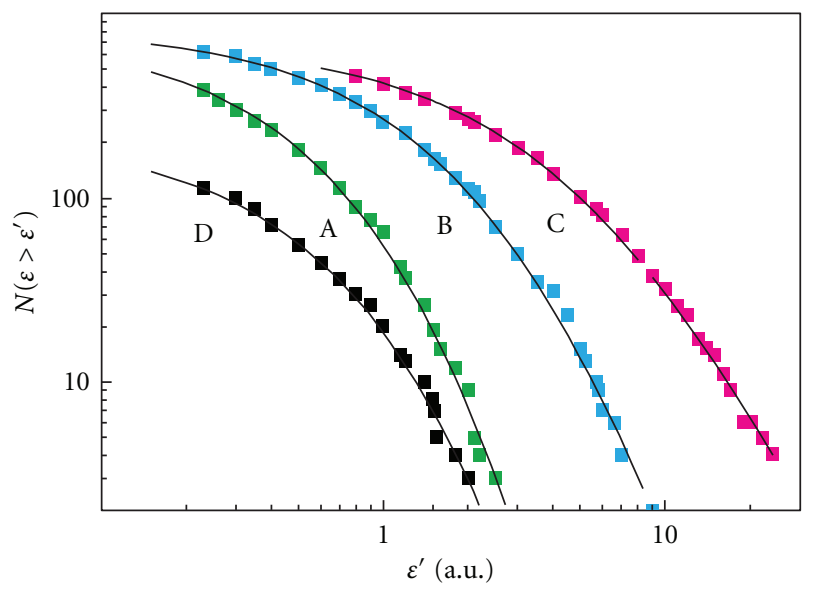

Figure 5: Experimental (signs) and calculated (curves) energy distributions $N\left(\varepsilon>\varepsilon^{\prime}\right)$ versus $\varepsilon^{\prime}$ in time windows A to D (see Figure 4) in the periods of time covering the icequakes in February 2004. The lines fit (8).

energy exchange between its components. It is the open character of the ASIC that causes nonlinear, synergic effects, such as the fractal geometry of lead-and-crack pattern. The flow of the external energy through the system increases the correlation radius of the event cross impact. The effect of local perturbations decays as the power law with covering much more significant neighborhood than that in the case of exponential decay inherent in the system under equilibrium condition. The principle of independence of individual events becomes disturbed, and the system exhibits a trend to selforganization.

Intuitively, one could expect that the scaling would be more pronounced under more nonequilibrium conditions. However, during a long time after the discovery of fractals by Mandelbrot no quantitative parameters were suggested for assessing the deviation of a dynamic system from its equilibrium state. Meanwhile, this problem grows out of abstract discussions because of its significance for forecasting the behavior of open natural systems. Apparently, the occurrence of natural hazards depends on the actual state of the given system. It appears that it is for this reason first attempts to estimate in numerical parameters the role of nonequilibrium state were made in seismology $[5,6,16]$.

In a similar way, the parameter of nonextensivity could serve as a characteristics of the state of drifting sea ice, which would provide information on potential changes in the pack motion and connectedness.

The example given in this communication demonstrates the drift dynamics of an individual ice floe during a substantial ice pack fragmentation, which had catastrophic subsequences for the research camp established on the pack. The terminal stage of the floe fracturing was preceded by the steady rise of the degree of nonequilibrium. A similar scenario one could expect for any object of marine engineering, which undergoes irregular impacts from the drifting ice. A response of the sea ice fragmentation on fluctuations in the thermodynamic state of the ice pack (equilibrium/nonequilibrium) contain a certain potential for forecasting icequakes in the Arctic region.

\section{Conclusion}

Multilevel fractal structures, such as the crack-and-lead pattern of fragmented sea ice, are formed in open dynamic systems driven by the external forcing. The statistical analysis of the drift dynamics in the Arctic Ocean in the period of time covering a large-scale ice pack fragmentation allowed us to assess the fluctuations in the thermodynamic state of the mobile sea ice. It was revealed that the most significant sea ice fragmentation characterized by the strongest fluctuations in the ice floe kinetic energy occurred in highly nonequilibrium state of the pack. The deviation of the drifting pack from its equilibrium state could be regarded as a precursor of forthcoming icequakes.

\section{References}

[1] B. Erlingsson, "Two-dimensional deformation patterns in sea ice," Journal of Glaciology, vol. 34, no. 118, pp. 301-308, 1988.

[2] A. Chmel, V. N. Smirnov, and L. V. Panov, "Characterization of the sea-ice floes issuing from their fractal properties," International Journal of Offshore and Polar Engineering, vol. 13, no. 1, pp. 128-132, 2003.

[3] C. Tsallis, Introduction to Nonextensive Statistical Mechanics-Approaching a Complex World, Springer, New York, NY, USA, 2009

[4] C. Tsallis, "The nonadditive entropy Sq and its applications in physics and elsewhere: some remarks," Entropy, vol. 13, pp. 1765-1804, 2011.

[5] L. Telesca, "Nonextensive analysis of seismic sequences," Physica A, vol. 389, no. 9, pp. 1911-1914, 2010.

[6] F. Vallianatos and P. Sammonds, "Is plate tectonics a case of non-extensive thermodynamics?" Physica A, vol. 389, no. 21, pp. 4989-4993, 2010.

[7] C. J. Keylock, "Describing the recurrence interval of extreme floods using nonextensive thermodynamics and Tsallis statistics," Advances in Water Resources, vol. 28, no. 8, pp. 773-778, 2005.

[8] A. Chmel, V. N. Smirnov, and L. V. Panov, "Scaling aspects of the sea-ice-drift dynamics and pack fracture," Ocean Science, vol. 3, no. 2, pp. 291-298, 2007.

[9] J. R. Marco and R.E. Thompson, "Rectilinear leads and internal motions in the ice pack of the Western Arctic Ocean," Journal of Geophysical Research, vol. 82, pp. 979-987, 1977.

[10] D. A. Rothrock and A. S. Thorndike, "Measuring the sea-ice floe size distribution," Journal of Geophysical Research, vol. 89, no. 4, pp. 6477-6486, 1984.

[11] M. L. Matsushita, "Fractal viewpoint of fracture and accretion," Journal of the Physical Society of Japan, vol. 54, no. 3, pp. 857-860, 1985.

[12] S. Abe and N. Suzuki, "Law for distance between successive earthquakes," Journal of Geophysical Research, vol. 33, pp. 8733-8738, 2003.

[13] S. Abe and N. Suzuki, "Scale-free statistics of time interval between successive earthquakes," Physica A, vol. 350, no. 2-4, pp. 588-596, 2005. 
[14] A. Chmel, V. N. Smirnov, and I. Shcherbakov, "Hierarchy of non-extensive mechanical processes in fracturing sea ice," Acta Geophysica, vol. 60, no. 3, pp. 719-739, 2012.

[15] A. Chmel, V. N. Smirnov, and I. B. Sheikin, "Variability of scaling time series in the Arctic sea-ice drift dynamics," Ocean Science, vol. 6, no. 1, pp. 211-217, 2010.

[16] Y. F. Contoyiannis and K. Eftaxias, "Tsallis and Levy statistics in the preparation of an earthquake," Nonlinear Processes in Geophysics, vol. 15, no. 3, pp. 379-388, 2008. 

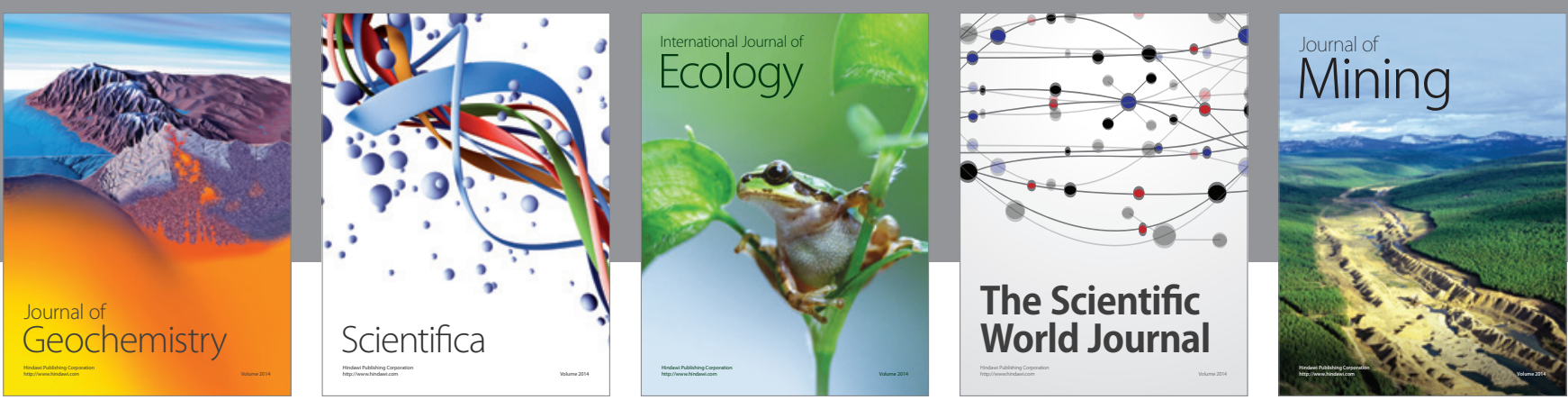

The Scientific World Journal
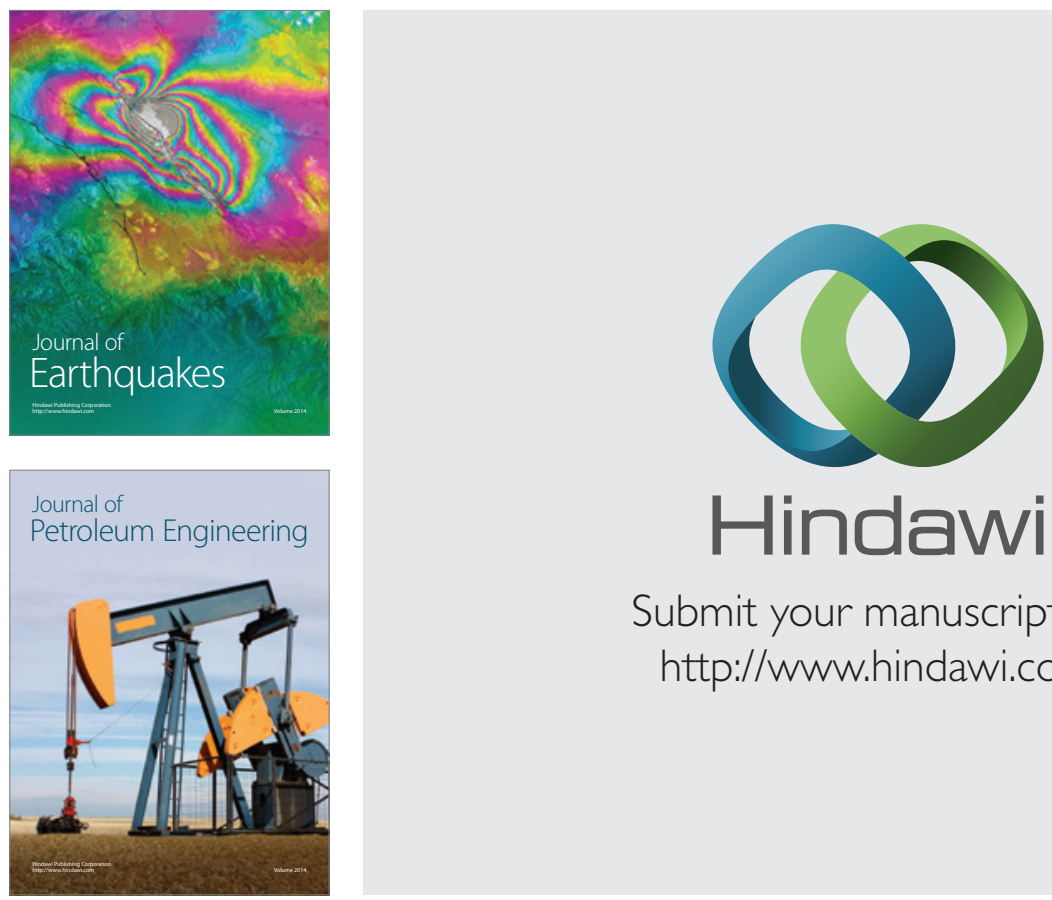

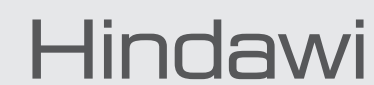

Submit your manuscripts at

http://www.hindawi.com
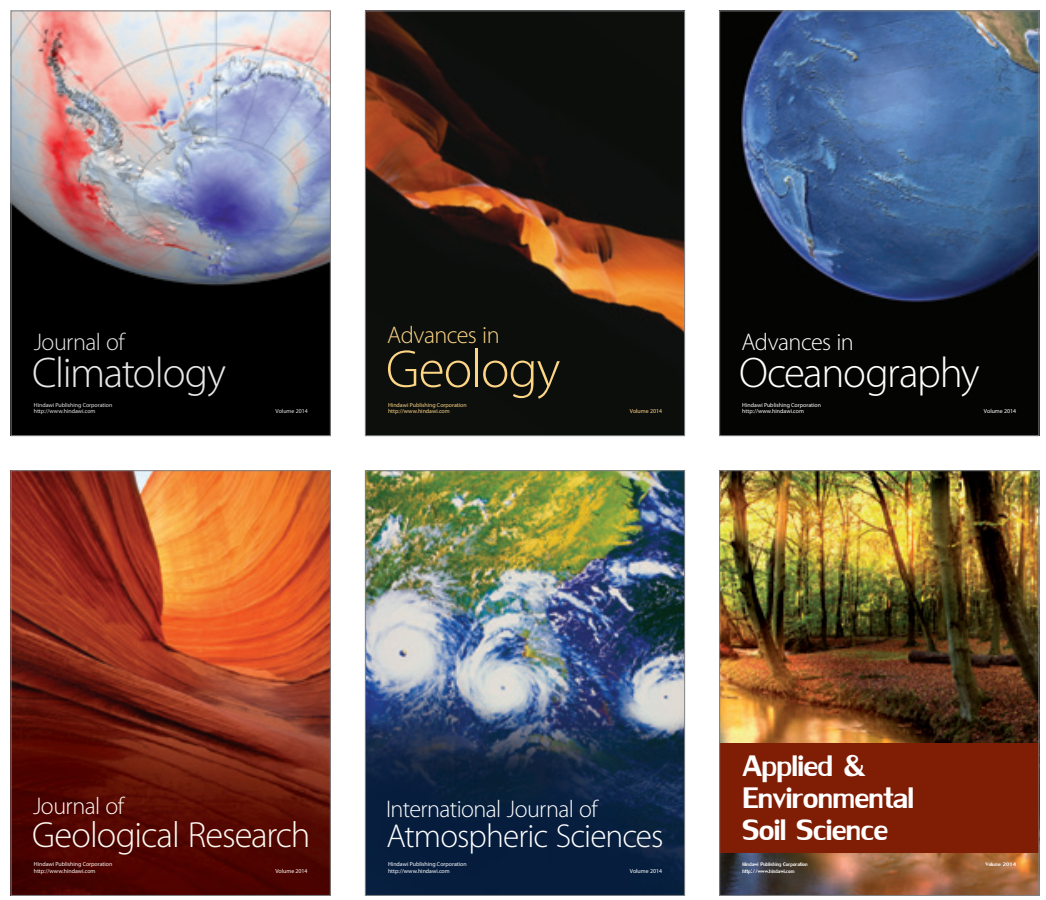
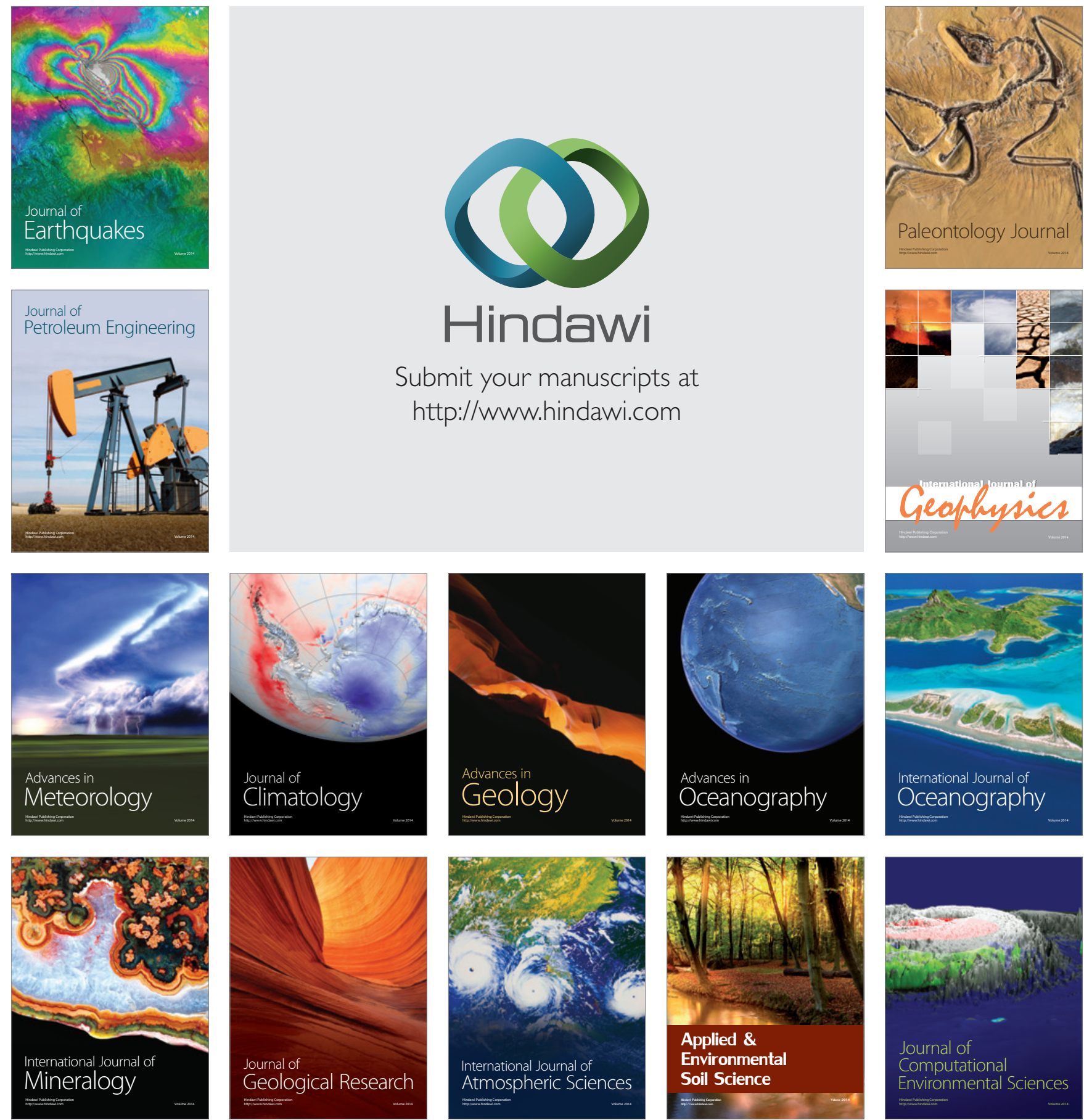